\title{
Is Charge Reduction in ESI Really Necessary?
}

\author{
Lloyd M. Smith \\ Department of Chemistry, University of Wisconsin-Madison, Madison, Wisconsin, USA
}

The technique of charge reduction electrospray mass spectrometry (CREMS), which can reduce the charge state complexity produced in electrospray ionization (ESI), is discussed. (J Am Soc Mass Spectrom 2008, 19, 629-631) (C) 2008 American Society for Mass Spectrometry

C harge reduction in the gas-phase occurs whenever oppositely charged ions combine. It has been in routine use in the atmospheric science community for particle analysis applications for decades [1]. In the last decade it has been developed in the field of biological mass spectrometry in two primary forms: at reduced pressure, typically inside of an ion trap mass spectrometer [2], and at atmospheric pressure, to reduce the charge of electrospray-generated ions before their entry into a mass spectrometer [3-6]. The latter approach has been developed primarily by our group at the University of Wisconsin-Madison, and it will serve as the principal subject of the present discussion.

We coined the acronym CREMS, for charge reduction electrospray mass spectrometry, for the approach. The nominal rationale for developing CREMS was to achieve complexity reduction in mass analyzing mixtures of many different molecules. The two main methods for producing gas-phase ions from macromolecules (e.g., proteins, nucleic acids, synthetic polymers) are matrix-assisted laser desorption/ionization (MALDI) and electrospray ionization (ESI). For reasons that are not yet entirely clear [7], MALDI produces primarily singly charged ions, whereas ESI produces a distribution of multiply charged ions in various charge states. Thus, whereas MALDI produces relatively simple mass spectra from mixtures of different molecules, with one major peak per analyte species, an ESI analysis of the same mixture may give an unresolved composite of many overlapping peaks. Charge reduction offered the potential of being able to have the simplicity of MALDI spectra while maintaining the advantages of ESI with respect to its real-time capability and gentler ionization.

We have developed two approaches to CREMS, differing in how reagent ions are generated. In the first approach [3, 4], we employed a ${ }^{210}$ Po source of $\alpha$-particles to create "air ions" in a chamber placed between the ESI capillary and the mass spectrometer inlet. We

Address reprint requests to Dr. L. M. Smith, Department of Chemistry, University of Wisconsin, 1101 University Avenue, Madison, WI 53706-1396, USA. E-mail: smith@chem.wisc.edu were able to show effective charge reduction of ESI-generated ions down to the singly charged state, with the desired reduction in charge state complexity and concomitant expansion of the $\mathrm{m} / \mathrm{z}$ range (lower $\mathrm{z}=>$ greater $\mathrm{m} / \mathrm{z}$ ). This yields "MALDI-like" ESI mass spectra from mixtures of proteins or nucleic acids. Figure 1 shows an example of the spectral simplification afforded by CREMS on a mixture of seven proteins (reproduced from reference [4] with permission). In subsequent work, we were able to employ a corona discharge source to produce reagent ions in place of the ${ }^{210}$ Po source $[5,6]$. This eliminated the regulatory and practical issues associated with obtaining and using radioactive materials, and in our most recent design the corona reduction chamber is quite small, efficient, and easy to put together [6].

Now, that is all a very nice background, but it does not address the question posed in the title of this article: "Is Charge Reduction Necessary?" This begs the question "Necessary for what?" What would one like to do with charge reduction that is not otherwise possible? Charge reduction might arguably find utility anytime one seeks to determine the masses of high molecular weight components in a mixture. Nucleic acids, synthetic polymers, and proteins are all compound classes of interest. Let us focus on proteins, as proteome analysis (a.k.a. proteomics) is the most active emerging area in mass spectrometry.

Proteomic analyses fall into two major bins: identification and quantification. For identification, the question is "What proteins are present in this sample?" For quantification, the question is "How much of each is present?" Both questions are usually addressed by means of peptide intermediaries. To find out what proteins are present in a sample, one typically digests the sample with trypsin to obtain the tryptic peptides, separates them by LC if necessary, and then either from the peptide masses or, more commonly, from the MS/MS fragmentation patterns, identifies the peptide [8]. The presence of the corresponding protein is inferred from the presence of peptides present in the protein sequence, and the more such peptides are identified for a given protein, the greater the confidence 
(a)

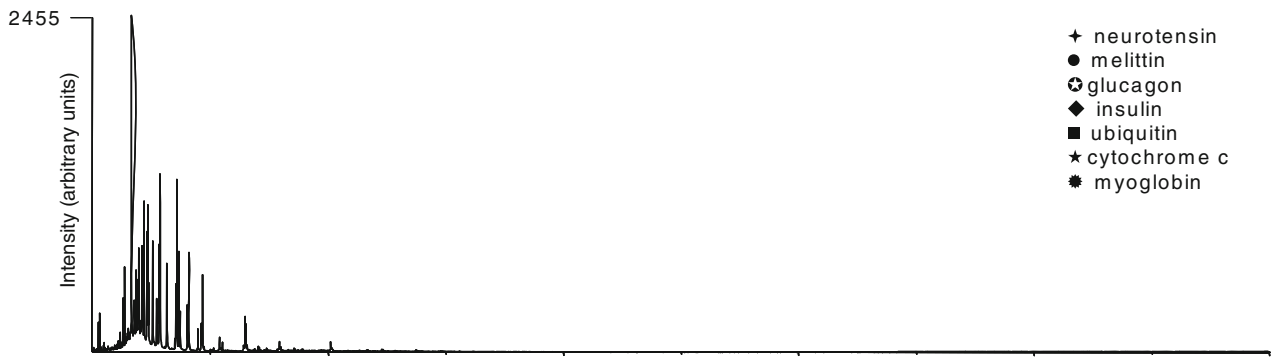

(b)

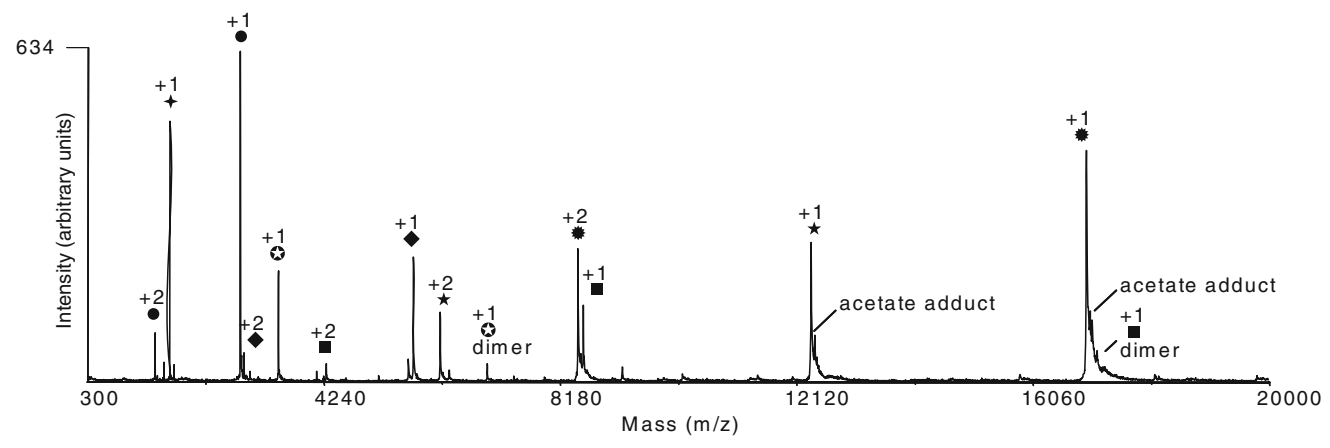

Figure 1. ESI spectra of an equimolar mixture of seven proteins (5 $\mu \mathrm{M}$ each in $1: 1 \mathrm{H}_{2} \mathrm{O} / \mathrm{CH}_{3} \mathrm{CN}, 1 \%$ acetic acid) without (a) and with (b) charge reduction. Adapted from reference [4] with permission. that the protein was in fact present. Quantification proceeds similarly, in that the amount of a given protein of interest is determined either from the absolute signal intensities for peptides of interest [9], or from their intensities relative to isotopically-tagged peptides used either as standards [10], or using heavy-light isotopic tagging chemistries [11].

This is all well and good, and these strategies constitute the major paradigm of our day for proteome analyses. However, there are some embarrassingly important aspects of proteomics that are not captured by these approaches. The most notable issue has to do with protein variation.

The ability to determine the extent and nature of protein variation is a critical missing piece in proteomics today. A surprise revealed by the success of the human genome project was the much lower than anticipated number of genes present in humans, in the range of $\sim 30,000$ rather than the predicted $\sim 100,000$ [12]. This fact has led to the general recognition that much of the complexity and sophistication afforded by our biological machinery is at the level of protein variation rather than just resulting from a large number of distinct genes [13]. These protein variations occur at three levels at least: alternative splicing of the RNA transcript, codon substitutions, and a wide variety of post-translational modifications. Such modified proteins play central roles in a wide variety of biological processes, from cellsignaling and signal transduction to gene regulation to controlling the activity of complex protein machines such as the ribosome or proteasome. For example, recent work in the identification of the isoforms of histone H3.2 has shown the existence of over 150 different post-translationally modified forms of the protein [14], and a similar analysis of histone $\mathrm{H} 4$ has revealed 74 different isoforms [15]. The levels of the many protein variants are dynamically regulated during the cell cycle and control the patterns of gene expression during development and differentiation.

Today, there is no technology available that can rapidly determine the nature and amounts of such protein variants present in a sample of interest. It is clear, however, that one is needed. In principle, charge reduction could play an important role in such an analysis. A plausible scenario is as follows: a sample of interest (imagine a nuclear extract) is subjected to an enrichment step for a set of proteins of interest (imagine immunoprecipitating histone $\mathrm{H} 3$ proteins). This moderate complexity sample is either directly infused into a mass spectrometer via electrospray and charge reduction, or subjected to a prior chromatographic or electrophoretic separation for further complexity reduction, followed by electrospray and charge reduction. In a wonderful world, every protein form would be represented by a peak in the mass spectrum, the intensity of that peak would reflect the relative amount of that species, and the width of the peak would be sufficiently narrow to allow that isoform to be identified (of course, such simple mass analysis would not provide any information on isomeric variants with identical masses). This might arguably also provide a useful tool for studying variations in protein complexes, as in low charge states there is much less electrostatic repulsion between the protein components, providing greater stability to the complex in the gas-phase.

This is a nice idea. What are the obstacles standing in the way of its implementation? There are quite a few. Variations in ionization efficiency and ionization sup- 
pression effects compromise the desired quantitative nature of the analysis [16]. Singly charged ions are more prone to adduction than highly charged ions, and such adducts can compromise mass analysis. The mass accuracy that can be obtained for large singly charged proteins is poor; the high $\mathrm{m} / \mathrm{z}$ range necessitates a time-of-flight (TOF) mass analyzer, and neither MALDI-TOF nor ESI-TOF are currently able to give resolutions in the dalton range (sufficient to discern the isotopic envelope) for mixtures of proteins in the 10,000 to 100,000 Da range. Ion detectors used in TOF analysis are generally based on secondary electron generation [electron multipliers, microchannel plates (MCPs)], the efficiency of which falls off strongly with increasing $\mathrm{m} / \mathrm{z}$ [17]. Thus, much work is needed on all aspects of large molecule mass spectrometry, including ionization processes, mass analyzer design, and detector performance, to make this scenario into a reality. Although clearly a difficult and long-term problem, I would argue that the importance of being able to open a window into this critical domain of proteomics is such that developing approaches to tackle these issues is an exciting challenge for the entire community. Of course, the approach outlined above is just one possible strategy, and doubtless many others could be conceived that would also merit exploration.

Are there any less daunting applications for charge reduction? The answer is likely yes. One interesting idea is to try to concentrate ions into single higher charge states, an approach pioneered by McLuckey using ion-ion reactions in ion traps, and termed "ion parking" [18]. If one were able to do this under the atmospheric pressure conditions employed in CREMS, there would be fewer issues with adduction (due to the higher charge states, which are easier to free of adducts), and the $m / z$ range would be reduced, possibly permitting other mass analyzers such as FTICR to be employed, with their concomitant advantages [19]. It would also help with ion detection, as highly charged ions are more easily detected, either directly (the greater number of charges leads to higher signal in Faraday cup or inductive detectors), or indirectly (as more highly charged ions acquire greater kinetic energy when accelerated by an electric field, and thus are more efficient in the generation of secondary electrons in electron multipliers or MCPs). One approach to this might be to employ labeling chemistry that converts specific protein functional groups (e.g., carboxylic acids) to fixed charges, as we and others have shown for metabolomics applications [20-22]. This could increase ionization efficiency, ideally to near unity, which would not only increase detection sensitivity, but would also greatly facilitate quantitation.

So, what is the response to the question "Is charge reduction in ESI really necessary?" Charge reduction has not yet enabled a critical application in mass spectrometric analysis. Exploiting its full potential will require significant advances in several other aspects of mass spectrometry, which may yet be many years from reality. However, the overriding problem it seeks to address, of deciphering the complexity of the proteome at the whole protein level, is a crucial unaddressed problem in biomolecular analysis. Whether by charge reduction or by some other approach, the development of solutions to this interesting and complex analytical challenge is an important priority for the community to address.

\section{References}

1. Adachi, M.; Okuyama, K.; Kousaka, Y.; Moon, S. W.; Seinfeld, J. H. Facilitated Aerosol Sizing Using the Differential Mobility Analyzer. Aerosol Sci. Technol. 1990, 12, 225-239.

2. Pitteri, S. J.; McLuckey, S. A. Recent Developments in the Ion/Ion Chemistry of High-Mass Multiply Charged Ions. Mass Spectrom. Rev. 2005, 24, 931-958.

3. Scalf, M.; Westphall, M. S.; Smith, L. M. Charge Reduction Electrospray Mass Spectrometry. Anal. Chem. 2000, 72(1), 52-60.

4. Scalf, M.; Westphall, M. S.; Krause, J.; Kaufman, S. L.; Smith, L. M. Controlling Charge States of Large Ions. Science 1999, 283, 194-197.

5. Ebeling, D. D.; Westphall, M. S.; Scalf, M.; Smith, L. M. Corona Discharge in Charge Reduction Electrospray Mass Spectrometry. Anal. Chem. 2000, 72(21), 5158-5161.

6. Frey, B. L.; Lin, Y.; Westphall, M. S.; Smith, L. M. Controlling Gas-Phase Reactions for Efficient Charge Reduction Electrospray Mass Spectrometry of Intact Proteins. J. Am. Soc. Mass Spectrom. 2005, 5, 1876-1887.

7. Karas, M.; Gluckmann, M.; Schafer, J. Ionization in Matrix-Assisted Laser Desorption/Ionization: Singly Charged Molecular Ions are the Lucky Survivors. J. Mass Spectrom. 2000, 35, 1-12.

8. Wolters, D. A.; Washburn, M. P.; Yates, J. R. An Automated Multidimensional Protein Identification Technology for Shotgun Proteomics. Anal. Chem. 2001, 73(23), 5683-5690.

9. Wang, W. X.; Zhou, H. H.; Lin, H.; Roy, S.; Shaler, T. A.; Hill, L. R.; Norton, S.; Kumar, P.; Anderle, M.; Becker, C. H. Quantification of Proteins and Metabolites by Mass Spectrometry Without Isotopic Labeling or Spiked Standards. Anal. Chem. 2003, 75(18), 4818-4826.

10. Gerber, S. A.; Rush, J.; Stemman, O.; Kirschner, M. W.; Gygi, S. P. Absolute Quantification of Proteins and Phosphoproteins from Cell Lysates by Tandem MS. Proc. Natl. Acad. Sci. U.S.A. 2003, 100(12), 6940-6945.

11. Gygi, S. P.; Rist, B.; Gerber, S. A.; Turecek, F.; Gelb, M. H.; Aebersold, R. Quantitative Analysis of Complex Protein Mixtures Using IsotopeCoded Affinity Tags. Nature Biotech. 1999, 17(10), 994-999.

12. Claverie, J.-M. What if There are Only 30,000 Human Genes. Science 2001, 291(5507), 1255-1257.

13. Stamm, S; Ben-Ari, S.; Rafalska, I.; Tang, Y. S.; Zhang, Z. Y.; Toiber, D.; Thanaraj, T. A.; Soreq, H. Function of Alternative Splicing. Gene 2005, $344,1-20$.

14. Garcia, B. A.; Pesavento, J. J.; Mizzen, C. A.; Kelleher, N. L. Pervasive Combinatorial Modification of Histone H3 in Human Cells. Nat. Methods 2007, 4(6), 487-489.

15. Phanstiel, D.; Brumbaugh, J.; Berggren, W. T.; Conard, K.; Feng, X.; Levenstein, M. E.; McAlister, G. C.; Thomson, J. A.; Coon, J. J. Mass Spectrometry Identifies and Quantifies 74 Unique Histone H4 Isoforms in Differentiating Human Embryonic Stem Cells. Proc. Nat. Acad. Sci. U.S.A., in press.

16. Taylor, P. J. Matrix Effects: The Achilles Heel of Quantitative HighPerformance Liquid Chromatography-Electrospray-Tandem Mass Spectrometry. Clin. Biochem. 2005, 38(4), 328-334.

17. Chen, X.; Westphall, M. S.; Smith, L. M. Mass Spectrometric Analysis of DNA Mixtures: Instrumental Effects Responsible for Decreased Sensitivity with Increasing Mass. Anal. Chem. 2003, 75(21), 5944-5952.

18. McLuckey, S. A.; Reid, G. E.; Wells, J. M. Ion Parking During Ion/Ion Reactions in Electrodynamic Ion Traps. Anal. Chem. 2002, 74(2), 336346.

19. Marshall, A. G. Milestones in Fourier Transform Ion Cyclotron Resonance Mass Spectrometry Technique Development. Int. J. Mass Spectrom. 2000, 200, 331-356.

20. Lamos, S. M.; Shortreed, M. R.; Frey, B. L.; Belshaw, P. J.; Smith, L. M. Relative Quantification of Carboxylic Acid Metabolites by Liquid Chromatography-Mass Spectrometry Using Isotopic Variants of Cholamine. Anal. Chem. 2007, 79(14), 5143-5149.

21. Yang, W. C.; Adamec, J.; Regnier, F. E. Enhancement of the LC/MS Analysis of Fatty Acids Through Derivatization and Stable Isotope Coding. Anal. Chem. 2007, 79(14), 5150-5157.

22. Krusemark, C. J.; Frey, B. L.; Belshaw, P. J.; Smith, L. M. Modifying the Charge State Distribution of Proteins in Electrospray Ionization Mass Spectrometry by Chemical Derivatization. 2007, unpublished. 